\title{
REVIEW
}

\section{Trabectedin for Soft Tissue Sarcoma: Current Status and Future Perspectives}

Erlinda M. Gordon · K. Kumar Sankhala · Neal Chawla •

Sant P. Chawla

Received: December 30, 2015 / Published online: May 27, 2016

(C) The Author(s) 2016. This article is published with open access at Springerlink.com

\section{ABSTRACT}

Trabectedin (ET743, Yondelis ${ }^{\circledR}$, manufactured by Baxter Oncology GmbH, Halle/Westfalen, Germany, for Janssen Products, LP, Horsham, PA), derived from the marine ascidian, Ecteinascidia turbinata, is a natural alkaloid with multiple complex mechanisms of action. On 23 October 2015, 15 years after the results of the first Phase 1 clinical trial using trabectedin for chemotherapy-resistant solid malignancies was reported, and 8 years after its approval in Europe, the United States Food and Drug Administration (USFDA) finally approved trabectedin for the treatment of unresectable or metastatic liposarcoma or leiomyosarcoma that has failed a prior anthracycline-containing regimen. Approval was based on the results of a pivotal Phase 3 trial involving a 2:1 randomization of 518 patients (who were further stratified by soft tissue sarcoma subtype), in which a significant

Enhanced content To view enhance content for this article go to http://www.medengine.com/Redeem/D1D 4F06053A388AB.

E. M. Gordon $(\bowtie) \cdot$ K. K. Sankhala · N. Chawla .

S. P. Chawla

Sarcoma Oncology Center/Cancer Center of

Southern California, Santa Monica, CA 90403, USA

e-mail: egordon@sarcomaoncology.com improvement in progression-free survival was reported in the trabectedin-treated group vs. the dacarbazine-treated group $(p<0.001)$. In this trial, the most common adverse reactions were nausea, fatigue, vomiting, constipation, anorexia, diarrhea, peripheral edema, dyspnea, and headache, while the most serious were neutropenic sepsis, rhabdomyolysis, cardiomyopathy, hepatotoxicity, and extravasation leading to tissue necrosis. The most common grade 3-4 adverse events were laboratory abnormalities of myelosuppression in both arms and transient transaminitis in the trabectedin arm. In a recent Phase 2 trial, trabectedin had a similar outcome as doxorubicin when given as a single agent in the first-line setting. Studies are also being conducted to expand the use of trabectedin not only as a first-line cancer drug, but also for a number of other clinical indications, for example, in the case of mesenchymal chondrosarcoma, for which trabectedin has been reported to be exceptionally active. The possibility of combining trabectedin with targeted therapies, immune checkpoint inhibitors or virotherapy would also be an interesting concept. In short, trabectedin is an 
old new drug with proven potential to impact the lives of patients with soft tissue sarcoma and other solid malignancies.

Funding: Sarcoma Oncology Center, Santa Monica, CA 90405.

Keywords: Alkylating agent; Clinical trials; Chemotherapy; DNA minor groove binder; ET-743; Oncology; Soft tissue sarcoma; Trabectedin

\section{OVERVIEW}

Soft tissue sarcoma is a rare tumor of the mesenchymal tissue, with many histological subtypes. It comprises about $1 \%$ of all adult cancers. The American Cancer Society estimated that in the USA in 2015, about 11,930 new cases would be diagnosed and 4870 Americans would die of soft tissue sarcomas [1]. The most common types of sarcoma in adults are undifferentiated pleomorphic sarcoma (formerly known as malignant fibrous histiocytoma), liposarcoma, and leiomyosarcoma. There is a tendency for certain types of sarcoma to originate from specific anatomic sites, such as the abdomen for leiomyosarcoma or the extremities for liposarcoma and undifferentiated pleomorphic sarcoma [2]. Surgical resection is the treatment of choice for localized disease, with radiation given as first-line therapy for unresectable cases. Nonetheless, $50 \%$ of high-grade tumors tend to recur [3]. For decades, treatment options for soft tissue sarcoma have been limited to doxorubicin and/or ifosfamide, and the outcome for metastatic disease is poor, with an estimated median survival of 8-13 months, as reported from results of randomized studies conducted over the last 20 years [4-7]. Targeted therapies have recently come of age for soft tissue sarcoma, with the
USFDA approval of pazopanib for locally advanced unresectable or metastatic soft tissue sarcoma with the exception of liposarcoma in 2012. Approval was based on the results of a randomized placebo-controlled Phase 3 (PALETTE) trial showing a significant, but modest, benefit in progression-free survival (PFS) for patients treated with pazopanib [8]. The approval of trabectedin in the USA in late 2015 shows promise for further improving the quality of life and progression-free survival of patients with soft tissue sarcoma.

Trabectedin is a natural alkaloid derived from the Caribbean tunicate, Ecteinascidia turbinata. It has multiple complex mechanisms of action [2, 9-13] and consequently the potential for extensive clinical applications. Further, several features of trabectedin's clinical performance differentiate it from other oncologic agents. These include prolonged tumor growth stabilization, favorable outcomes in sarcomas with genetic mutations, durability of responseeven upon treatment reinstitution after interruption of therapy, and absence of cumulative toxicity [14]. This article reports on the critical stages of drug development of trabectedin in the US and worldwide and provides perspectives on its future as a uniquely effective oncologic agent for soft tissue sarcomas and other solid malignancies.

This article is based on previously conducted studies and does not involve any new studies of human or animal subjects performed by any of the authors.

\section{CRITICAL STAGES OF TRABECTEDIN DRUG DEVELOPMENT}

\section{Preclinical Studies}

Trabectedin, derived from the marine tunicate, Ecteinascidia turbinata, has multiple complex mechanisms of action. In preclinical studies, 
trabectedin has been shown to bind to the N2 amino group of guanine residues in the minor groove of the DNA double helix and cause double-strand breaks $[2,9,10]$. Second, trabectedin interrupts the cell cycle, causes apoptosis of cancer cells and downregulates abnormal transcription factor expression such as FUS-CHOP or EWS-CHOP [10]. Third, trabectedin inhibits cytokine release by monocytes and macrophages in the tumor microenvironment via its direct cytotoxic effects on tumor-associated macrophages [11, 12]. This drug effect on the tumor microenvironment is deemed critically important in cancer therapy because of the resultant inhibition of neoangiogenesis and the metastatic potential of cancer cells [13].

\section{Clinical Studies}

\section{Efficacy Studies}

Table 1 lists selected Phase 1 clinical trials using trabectedin for advanced solid tumors and the Phase 2 and 3 studies for soft tissue sarcoma, including retrospective analytical reports and data from the expanded access program. There are more studies of trabectedin conducted for other clinical indications that are not listed in the tables.

\section{Phase 1 Studies}

The goal of the Phase 1 clinical trials, which involved patients with advanced solid malignancies, was to determine the dose-limiting toxicity and maximum tolerated dose of trabectedin as well as to evaluate its pharmacokinetics, pharmacodynamics, and potential for adverse drug reactions. There were at least seven reported Phase 1 studies using trabectedin as a single agent for advanced solid tumors [15-21] and five Phase 1 studies using trabectedin in combination with either doxorubicin, doxil, gemcitabine, or cisplatin (Table 1) [22-26].

In 2001, Delaloge et al. [16] first reported on the clinical activity of trabectedin in 29 patients with soft tissue sarcoma who had failed treatment with doxorubicin and one other chemotherapeutic agent (12 from a phase 1 trial and 17 from a compassionate use program cohort). In this study, there were 4/29 partial responses (PR), 2/29 minor responses with tumor reduction of at least $30 \%$ in both cases, and 10/29 stable disease (SD) lasting more than 2 months and median time to progression of 2.8 months. In the same year, Taama et al. [17] determined the optimal regimen of trabectedin to be $1.5 \mathrm{mg} /$ $\mathrm{m}^{2}$ as a 24-h continuous intravenous infusion once every 3 weeks from a Phase 1 study involving 52 patients. Trabectedin was characterized by a moderate plasma clearance (31.5 and $37.5 \mathrm{l} / \mathrm{h}$ in the absence and presence of coadministered dexamethasone, respectively) and a large volume of distribution at steady state (in excess of 5000 l) [27]. The biologic half-life of trabectedin ranged from 27 to $89 \mathrm{~h}$ in pharmacokinetic studies [15, 18, 19], depending on the mode of administration and infusion schedule. The terminal half-life calculated using data from 14 Phase 1 and Phase 2 studies using non-linear mixed effects models was longer, in the range of $175 \mathrm{~h}$ [27].

Of the five Phase 1 studies using trabectedin and one other chemotherapeutic agent, the most promising combination regimen in advanced soft tissue sarcomas (STS) and breast cancer was trabectedin with doxorubicin, with an overall response rate (ORR) of $18 \%$, SD of $56 \%$, and disease control rate (DCR) of $74 \%$ [26]. 


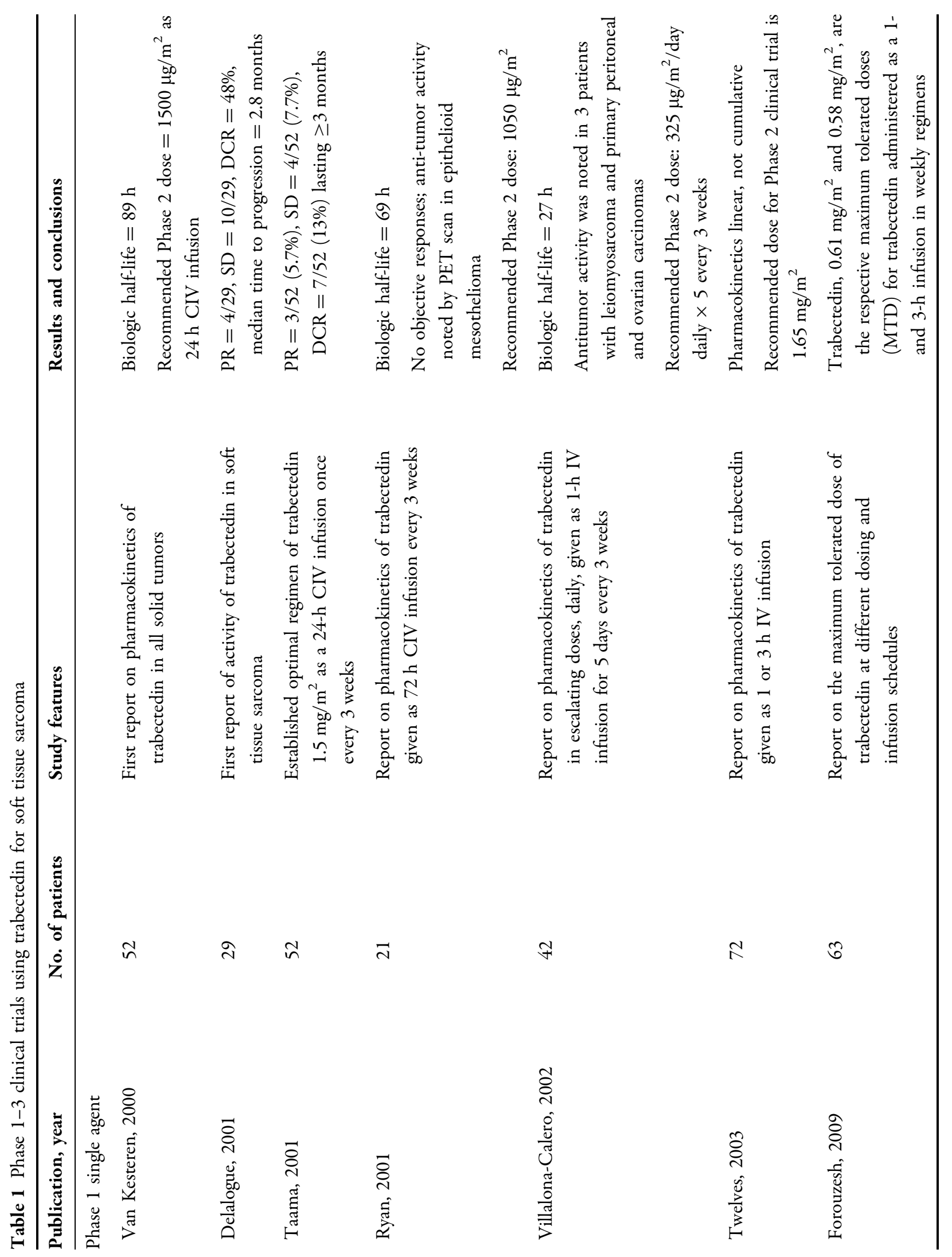




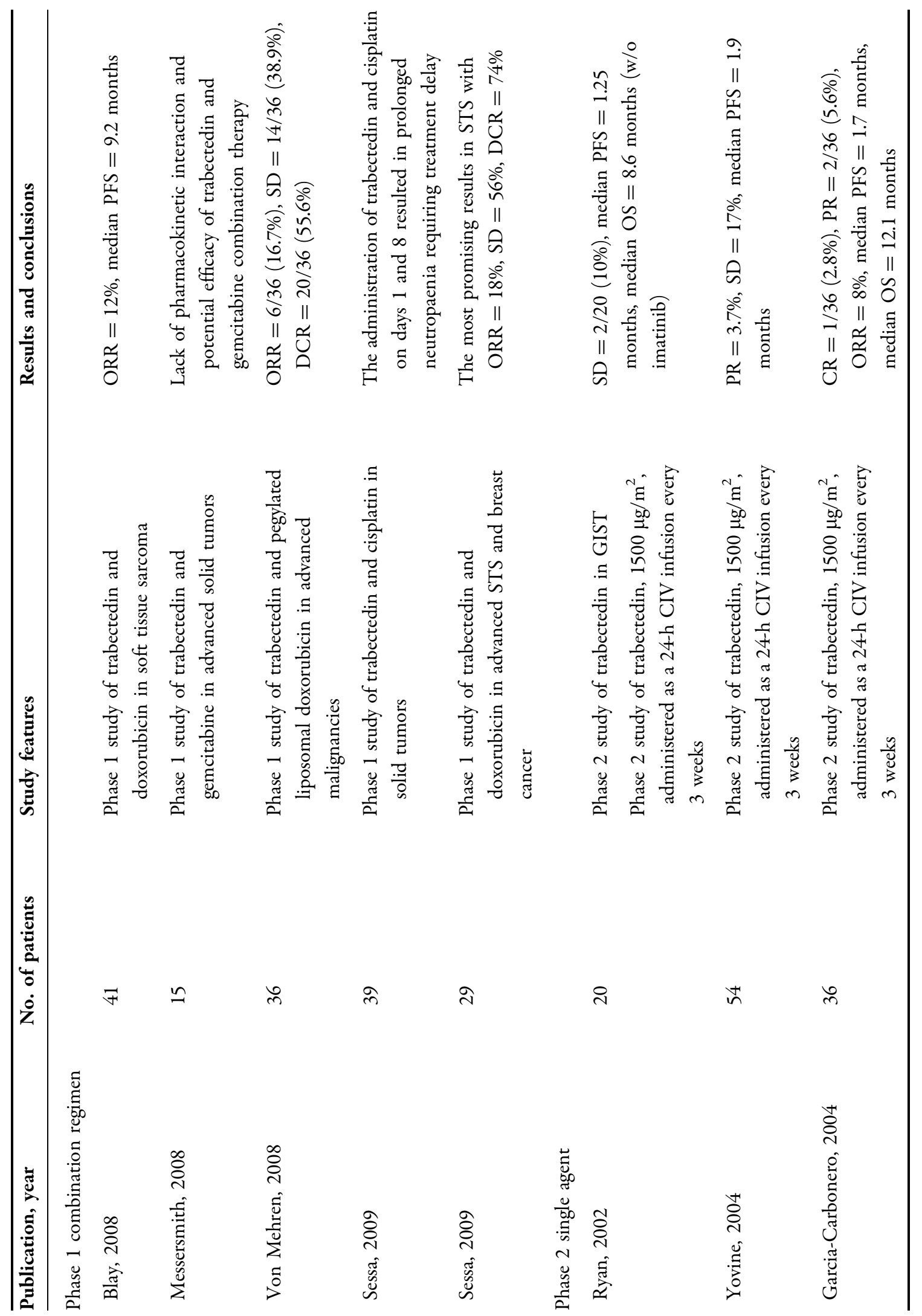




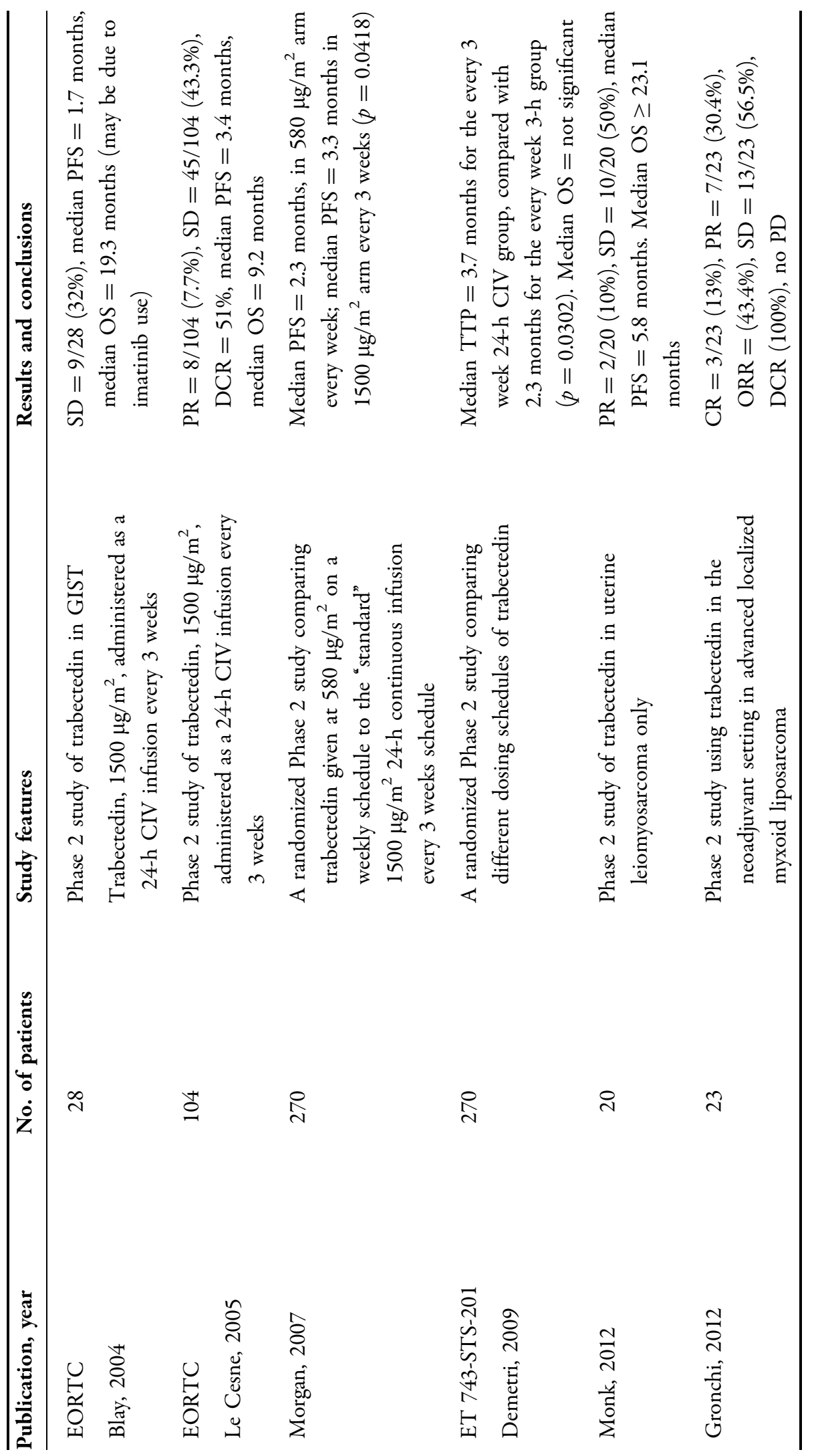




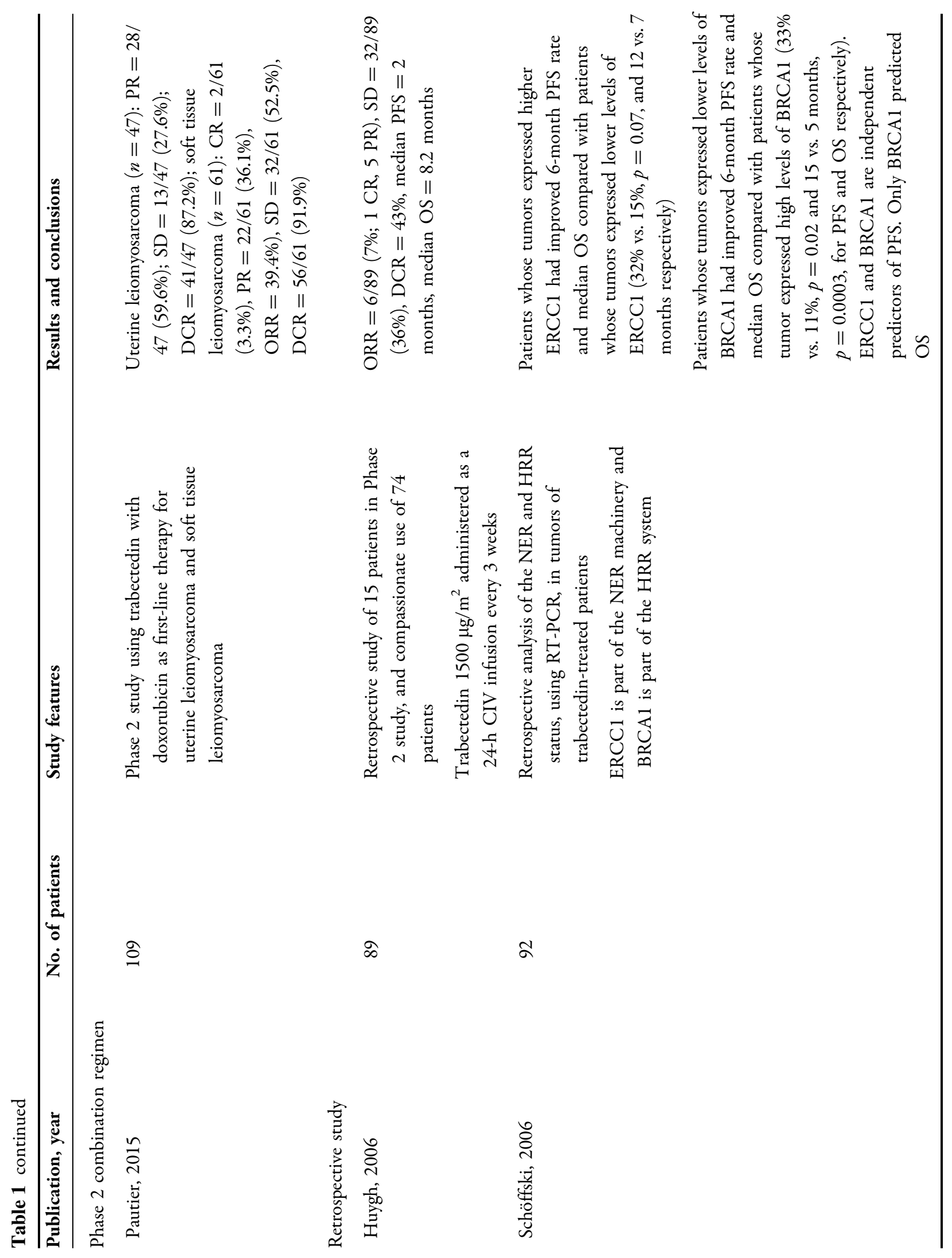




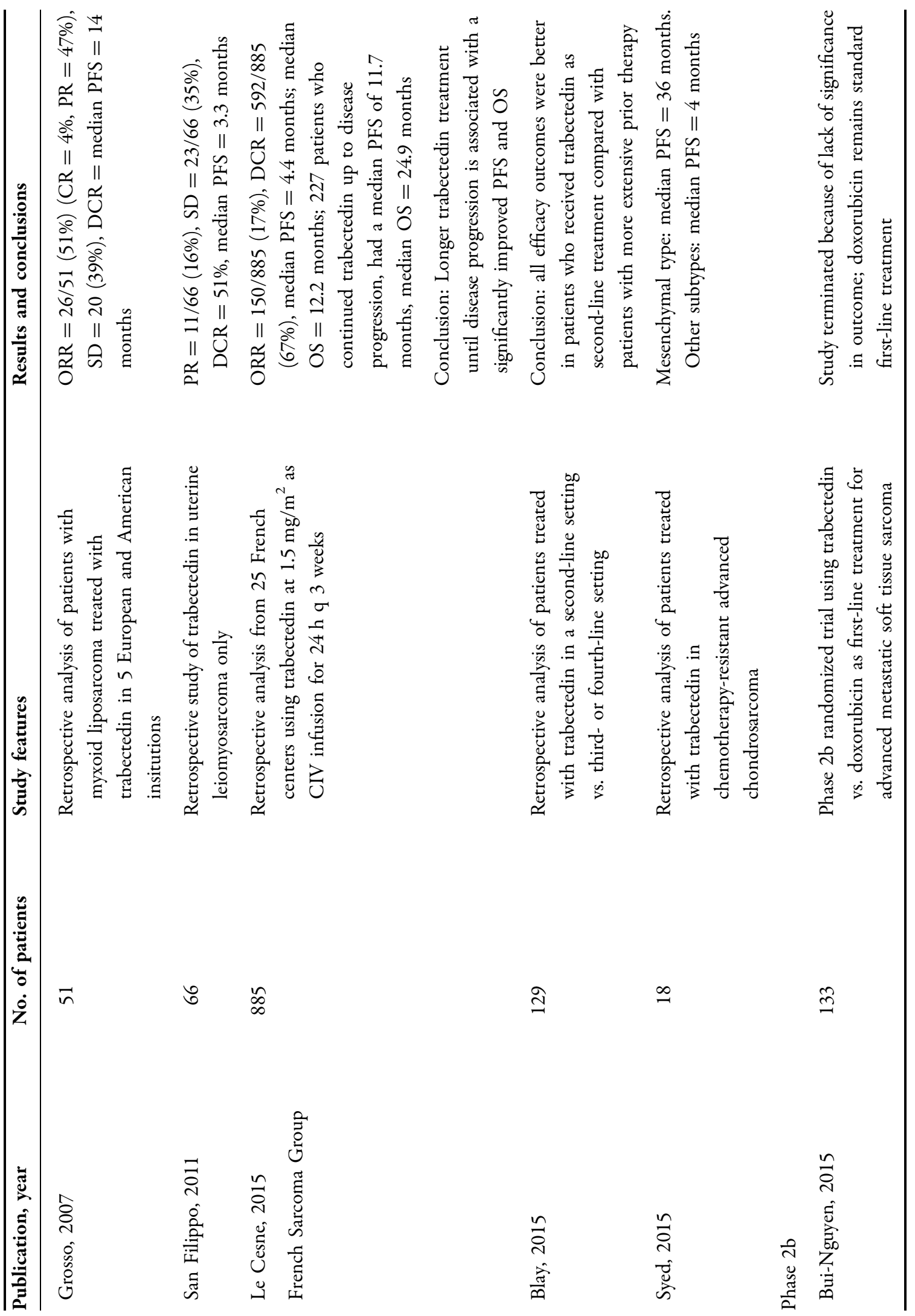




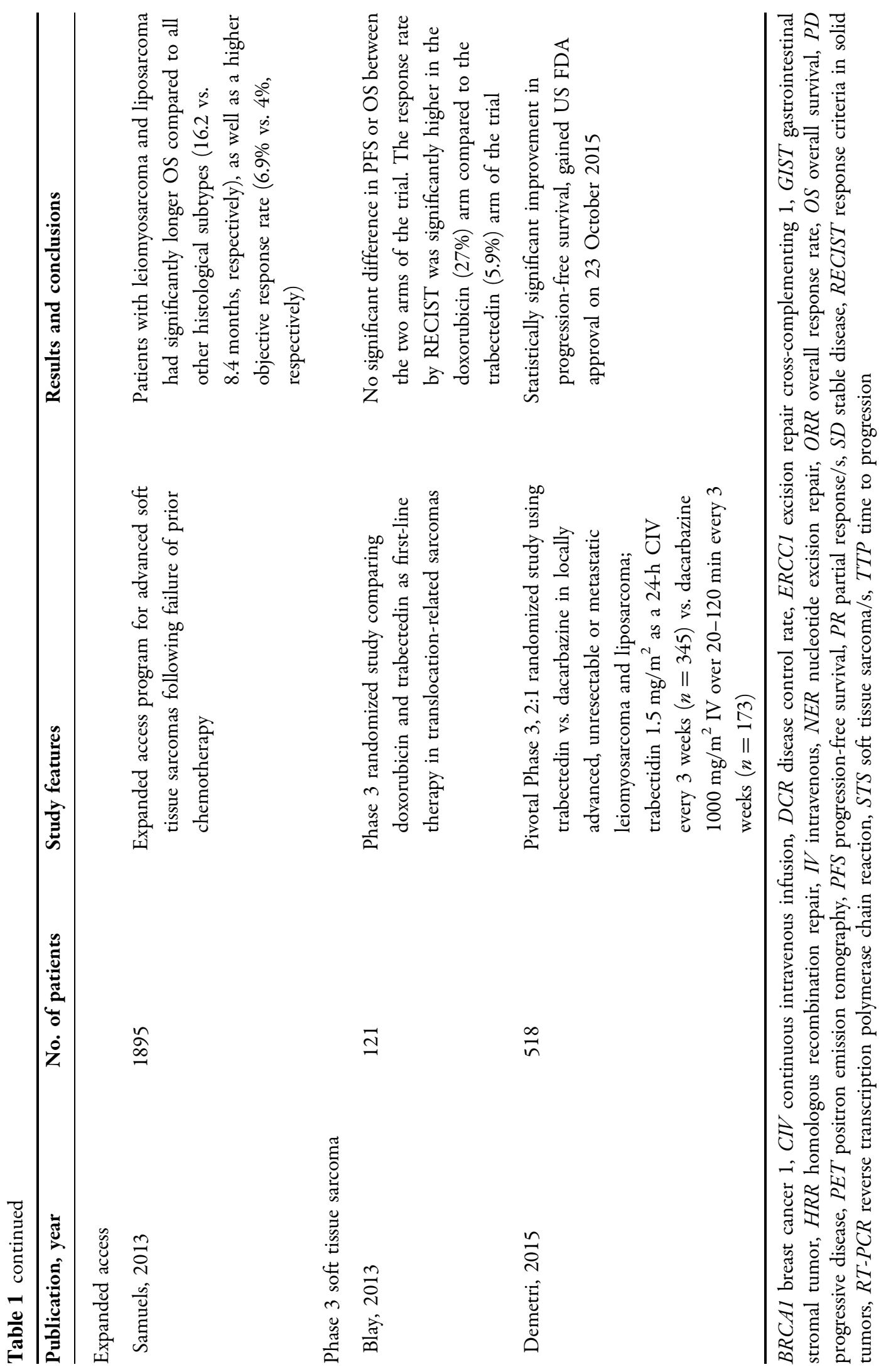




\section{Phase 2 Studies}

The goal of the Phase 2 clinical trials was to evaluate the safety and efficacy of trabectedin at the recommended dosage and mode of administration derived from the results obtained from Phase 1 trials in a larger number of patients with STS who have failed standard chemotherapy. Some investigators reported on the use of trabectedin in the first-line and the neoadjuvant settings. At least nine Phase 2 clinical studies have been conducted worldwide (Table 1) [28-36]. The efficacy and safety of trabectedin in soft tissue sarcoma are based on a randomized trial, STS-201, in patients with locally advanced or metastatic lipo- or leiomyosarcoma, whose disease had progressed or relapsed after treatment with at least anthracyclines and ifosfamide. Additionally, in 2005, Le Cesne and the EORTC [32] reported the results of a Phase 2 study using trabectedin at $1.5 \mathrm{mg} / \mathrm{m}^{2}$ CIV in 104 patients. In that study, there were 8 (7.7\%) PRs and 45 (43.3\%) SDs. After a median follow-up of 34 months, the median PFS was 3.4 months, and the median overall survival was 9.2 months. The results of these Phase 2 trials led to the accelerated approval of trabectedin by the European Union for advanced soft tissue sarcoma in 2007. The best Phase 2 results were reported by Monk et al. [34] in 2012, with a PFS of 5.8 months in patients with uterine leiomyosarcoma.

\section{Retrospective Studies}

At least five retrospective studies were conducted between 2006 and 2015 [37-41]. One study involved the analysis of 885 patients from 25 French centers using trabectedin at $1.5 \mathrm{mg} / \mathrm{m}^{2}$ as CIV infusion for $24 \mathrm{~h}$ every 3 weeks [41]. In this study, the reported ORR was $17 \%$, with a DCR of $67 \%$, PFS of 4.4 months, and median overall survival
(OS) of 12.2 months. In a report by Grosso et al. (2007) [39], retrospective analysis of patients with myxoid liposarcoma in five European and American institutions showed an ORR of 51\%, DCR of $90 \%$, and median PFS of 14 months. In 2011, San Filippo et al. [40] reported a PR of $16 \%$, SD of $35 \%$, and PFS of 3.3 months in patients with uterine leiomyosarcoma.

\section{Phase 3 Studies}

Two Phase 3 randomized clinical trials were conducted. One study compared the efficacy of trabectedin vs. doxorubicin in translocation-related sarcomas [42]. The other compared the PFS using trabectedin vs. dacarbazine [43].

The Phase 3 randomized study comparing doxorubicin and trabectedin as first-line therapy for translocation-related sarcomas enrolled 121 patients and reported no significant difference in PFS or OS between the two arms of the trial, with the response rate by response criteria in solid tumors (RECIST) significantly higher in the doxorubicin arm (27\%) compared to the trabectedin arm (5.9\%). Consequently, doxorubicin remains the first-line treatment for translocation-related sarcoma. It is important to note, however, that the study was underpowered because of the high censoring rate and high rate of ineligible patients.

In the pivotal Phase 3 study using trabectedin vs. dacarbazine in locally advanced, unresectable, or metastatic leiomyosarcoma and liposarcoma, patients were randomized at a 2:1 trabectedin:dacarbazine ratio. The study enrolled a total of 518 patients, with 345 randomized to the trabectedin arm and 173 to the dacarbazine arm [43]. Trabectedin was given at a dose of $1.5 \mathrm{mg} / \mathrm{m}^{2}$ as a 24-h CIV every 3 weeks and dacarbazine at $1000 \mathrm{mg} / \mathrm{m}^{2}$ IV over 20 to 120 min every 3 weeks. The median patient age 
was 56 years (range 17-81), and 30\% were male, $77 \%$ white, $12 \%$ black, and $4 \%$ Asian. The study was further stratified based on subtype (leiomyosarcoma vs. liposarcoma), Eastern Cooperative Oncology Group (ECOG) score (0 or 1 ), and number of previous chemotherapies (1 vs. 2 or more). Previous chemotherapy included doxorubicin and ifosfamide, or doxorubicin or ifosfamide and one other drug. Doxorubicin was used in $90 \%$ of cases, gemcitabine in $81 \%$, docetaxel in $74 \%$, ifosfamide in $59 \%$, and pazopanib in $10 \%$. Seventy-three percent of patients had leiomyosarcoma, and $27 \%$ had liposarcoma. Forty-nine percent had an ECOG score of 0 , and $89 \%$ had two prior chemotherapy regimens. Median PFS for trabectedin vs. dacarbazine was 4.2 and 1.5 months, respectively (hazard ratio, $0.55 ; p<0.001$ ). Based on a significant improvement in PFS for the trabectedin arm, the USFDA gave full marketing approval of trabectedin for leiomyosarcoma and liposarcoma on 23 October 2015.

\section{Expanded Access Program}

The expanded access program for advanced soft tissue sarcomas following failure of prior chemotherapy enrolled 1895 patients worldwide (Table 1) [44]. Analysis of the data revealed that patients with leiomyosarcoma and liposarcoma had a higher ORR (6.9\% vs. $4 \%$, respectively) and significantly longer OS (16.2 vs. 8.4 months, respectively) than all other histological subtypes. Patient enrollment has continued beyond this publication.

\section{Toxicity Studies}

The adverse events reported in the Phase 3 trial of trabectedin vs. dacarbazine in leiomyosarcoma and liposarcoma patients who had failed at least one anthracycline-based regimen and one of another chemotherapeutic agent are listed in the USFDA Product Information document [45]. The most common adverse reactions occurring in greater than $10 \%$ of patients, and at a higher incidence than the control arm receiving dacarbazine, include nausea in $75 \%$, fatigue in $69 \%$, vomiting in $46 \%$, constipation and decreased appetite in 37\%, and diarrhea in 35\%. Less common adverse reactions were dyspnea and headache in 25\%, arthalgia and insomnia in $15 \%$, and myalgia in $12 \%$. Grade $3-4$ adverse reactions were uncommon $(<10 \%)$. The most common laboratory abnormalities include anemia in 96\%, increased alanine aminotransferase (ALT), aspartate aminotransferase (AST), and alkaline phosphatase levels in 90, 84, and 70\%, respectively, neutropenia in $66 \%$, hypoalbuminemia in 63\%, thrombocytopenia in $59 \%$, increased creatine phosphokinase in $33 \%$, and hyperbilirubinemia in 13\%. Among the common grade $3-4$ adverse events ( $>10 \%)$ were neutropenia in $43 \%$, thrombocytopenia in $21 \%$, anemia in 19\%, and increased ALT and AST levels in $31 \%$ and $17 \%$, respectively.

An important factor in reducing toxicity is the use of dexamethasone as pre-medication before starting trabectedin infusion. In a previous study by Grosso et al., the incidence of grade 3-4 liver enzyme elevation, neutropenia, and thrombocytopenia fell to 3 , 10 , and $0 \%$, respectively, in patients who received routine antiemetic prophylaxis with steroids on day 0 and possibly on day +1 , compared with 70,39 , and 35\%, respectively, in the group who received dexamethasone prophylaxis $4 \mathrm{mg}$ PO BID the day before trabectedin infusion $(p=0.0001)[46,47]$. 


\section{TRABECTEDIN APPLICATIONS IN THE CLINIC}

In the European Union, trabectedin (Yondelis ${ }^{\circledR}$ manufactured by Baxter Oncology $\mathrm{GmbH}$, Halle/Westfalen, Germany, for Janssen Products, LP, Horsham, PA) gained marketing approval for ovarian cancer and soft tissue sarcoma under "exceptional circumstances" from the European Commission in September 2007 based on favorable results of Phase 2 studies [48]. The following is a summary of the European Public Assessment Report (EPAR) for trabectedin, which explained the process used by the Committee for Medicinal Products for Human Use (CHMP) in granting marketing approval and provided recommendations for optimum drug administration. For soft-tissue sarcoma, patients who received trabectedin at $1.5 \mathrm{mg} / \mathrm{m}^{2}$ every 3 weeks had an average of 3.8 months PFS compared with 2.1 months in patients who received a lower dose-three times per month. For ovarian cancer, patients who received the combination of trabectedin and pegylated liposomal doxorubicin (PLD) had a longer average PFS (7.3 months) compared to those patients who received PLD alone (5.8 months). In these Phase 2 studies, $10 \%$ of patients treated with trabectedin as a single agent and $25 \%$ treated with trabectedin in combination therapy had serious side effects. The most common side effects of any severity were neutropenia, nausea, vomiting, increase in liver enzymes, anemia, fatigue, thrombocytopenia, anorexia, and diarrhea. Trabectedin gained full marketing approval for ovarian cancer and soft tissue sarcoma from the European Commission in May 2015.

In the US, trabectedin (Yondelis,) gained FDA approval on 23 October 2015 for unresectable or metastatic liposarcoma or leiomyosarcoma patients who received a prior anthracycline-containing regimen [45]. The recommended dose is $1.5 \mathrm{mg} / \mathrm{m}^{2}$ administered as a continuous intravenous infusion over $24 \mathrm{~h}$ through a central venous line every 21 days in patients with normal bilirubin and AST or ALT $\leq 2.5$ times the upper limit of normal. Since there was no evidence of cumulative toxicity in the Phase 3 clinical trials, trabectedin may be given until disease progression or unacceptable toxicity occurs. There is no recommended dose of trabectedin in patients with serum bilirubin levels above the institutional upper limit of normal. The product information recommends premedication with $20 \mathrm{mg}$ dexamethasone intravenous over $30 \mathrm{~min}$ prior to each trabectedin dose to reduce documented liver toxicity $[45,46]$.

Based on the toxicity profile of trabectedin, the USFDA product information [45] recommends dose modifications for neutropenia, thrombocytopenia, elevated bilirubin, serum transaminases, and creatine phosphokinase, decreased left ventricular ejection fraction or clinical evidence of cardiomyopathy, or any grade 3 or 4 non-hematologic adverse reactions. The first recommended dose reduction is to $1.2 \mathrm{mg} / \mathrm{m}^{2}$ every 3 weeks, the second to $1.0 \mathrm{mg} / \mathrm{m}^{2}$ every 3 weeks. Once reduced, the dose of trabectedin should not be increased in subsequent treatment cycles. Recommended dose modifications include permanently discontinuing trabectedin for persistent adverse reactions requiring a delay in dosing of more than 3 weeks, for adverse reactions requiring dose reduction following trabectedin administered at $1.0 \mathrm{mg} / \mathrm{m}^{2}$, and for severe liver dysfunction in the prior treatment cycle.

Drug interactions can also occur between trabectedin and cytochrome CYP3A inhibitors or inducers. Therefore, the use of strong CYP3A 
inhibitors (e.g., oral ketoconazole, itraconazole, posaconazole, voriconazole, clarithromycin, telithromycin, indinavir, lopinavir, ritonavir, boceprevir, nelfinavir, saquinavir, telaprevir, nefazodone, and conivaptan) should be avoided in patients taking trabectedin. Grapefruit or grapefruit juice should also be avoided during trabectedin treatment, as well as the use of strong cytochrome CYP3A inducers such as rifampin, phenobarbital, and St. John's wort [45].

\section{THE FUTURE OF TRABECTEDIN}

In the European Union, trabectedin has been approved for ovarian cancer and soft tissue sarcoma. Given that USFDA approval of trabectedin is limited to only two subtypes of soft tissue sarcoma, i.e., unresectable or metastatic liposarcoma or leiomyosarcoma patients who received a prior anthracycline-containing regimen, trials for other types of sarcoma that respond well to trabectedin, such as mesenchymal chondrosarcoma, will provide additional data to support its use in the absence of an FDA-approved clinical indication.

Evidence to support the use of trabectedin in mesenchymal chondrosarcoma involves the reported positive results of a single-center retrospective analysis of patients treated in various IRB-approved trials [49]. The objective was to examine the clinical benefit of trabectedin to various chondrosarcoma subtypes in a cohort of patients with advanced unresectable disease. Patients included in this retrospective analysis received trabectedin administered at doses of $1.2-1.5 \mathrm{mg} / \mathrm{m}^{2}$ by $24-\mathrm{h}$ infusion every 3 weeks. Tumor response was evaluated from serial scans (computed tomography/magnetic resonance imaging/positron emission tomography) by
RECIST 1.1 criteria every 8 weeks. Treatment adverse effects were assessed by clinical evaluation and laboratory investigations.

Briefly, there were a total of 18 patients studied: 5 with conventional chondrosarcoma, 5 with mesenchymal chondrosarcoma, 5 with myxoid chondrosarcoma, and 3 with de-differentiated chondrosarcoma. Mean age was 53 years with a male-to-female ratio of 11:7. Adverse events were as follows: $33 \%$ of patients had CTCAE grade 3-4 thrombocytopenia at least once; $11 \%$ had grade 3-4 neutropenia not associated with febrile episodes; one patient had grade 3-4 anemia; one patient had grade 3-4 elevated liver enzymes; none developed cardiotoxicity or nephrotoxicity during treatment; one patient discontinued treatment because of port infiltration after one cycle. Treatment dosage was reduced in 10 of 18 (56\%) patients in response to adverse events and side effects.

Analyses of the safety and efficacy of trabectedin use in mesenchymal chondrosarcoma are as follows: (1) patients with mesenchymal chondrosarcoma had a higher median PFS and percentage PFS at 3 and 6 months than patients with other chondrosarcoma subtypes, (2) trabectedin has manageable hematological side effects and did not result in any cardiotoxicity or nephrotoxicity in our cohort, and (3) there was no evidence of cumulative toxicity even with prolonged duration of treatment.

Other promising studies include a recent report of trabectedin activity in patients with translocation-related sarcomas. In this Phase 2 open-label randomized study, trabectedin was compared with best supportive care as second or later line treatment. A total of 76 patients were enrolled with 73 evaluable patients. Median PFS was 5.6 months for trabectedin-treated patients 
vs. 0.9 months for patients who received best supportive care $(p<0.0001)$. These data suggest a significant increase in PFS in trabectedin-treated patients compared to those who received best supportive care [50]. A number of clinical trials are ongoing using combination regimens of trabectedin with olaparib [a poly (ADP-ribose) polymerase (PARP) inhibitor] or with radiotherapy [51]. Retrospective analysis of patients treated with trabectedin in second- and third-line settings shows a trend toward earlier treatment with trabectedin [52]. Prospective investigations could include combination regimens with trabectedin and promising chemotherapeutic agents, such as eribulin and aldoxorubicin. Cancer immunotherapy is also coming of age, and combination regimens of trabectedin-to expose tumor neoantigens in the TME-with immune checkpoint inhibitors and/or oncolytic viruses expressing granulocyte macrophage colony stimulating factor (GM-CSF) to recognize the neoantigens, may evoke a favorable immunologic response in certain patients with soft tissue sarcomas.

\section{ACKNOWLEDGMENTS}

The study was funded by the Sarcoma Oncology Center, Santa Monica, CA, 90405. All named authors meet the International Committee of Medical Journal Editors (ICMJE) criteria for authorship for this manuscript, take responsibility for the integrity of the work as a whole, and have given final approval for the version to be published.

The authors are grateful to Heather C. Gordon for editorial assistance in the writing of this manuscript.

EMG reviewed the medical literature, wrote the draft of the manuscript, created the table, reviewed and revised the manuscript. KKS reviewed the medical literature and reviewed and helped revise the manuscript. NSC reviewed the literature, reviewed and helped revise the manuscript. SPC reviewed the literature, reviewed and helped revise the manuscript. All authors read and approved the final manuscript. During the peer review process, the manufacturer of the agent under review was offered an opportunity to comment on the article. Changes resulting from comments received were made by the author based on their scientific and editorial merit.

Disclosures. E. M. Gordon, K. K. Sankhala, N. Chawla and S. P. Chawla have nothing to disclose.

Compliance with Ethics Guidelines. This article is based on previously conducted studies and does not involve any new studies of human or animal subjects performed by any of the authors.

Open Access. This article is distributed under the terms of the Creative Commons Attribution-NonCommercial 4.0 International License (http://creativecommons.org/licenses/ by-nc/4.0/), which permits any noncommercial use, distribution, and reproduction in any medium, provided you give appropriate credit to the original author(s) and the source, provide a link to the Creative Commons license, and indicate if changes were made.

\section{REFERENCES}

1. American Cancer Society at http://www.cancer.org/ acs/. Accessed 28 Dec 2015.

2. Petek BJ, Loggers ET, Pollack SM, Jones RI. Trabectedin in soft tissue sarcomas. Mar Drugs. 2015;13:974-83. 
3. Clark MA, Fisher C, Judson I, Thomas JM. Soft-tissue sarcomas in adults. $\mathrm{N}$ Engl J Med. 2005;353:701-11.

4. Antman K, Crowley J, Balcerzak SP, et al. An intergroup phase III randomized study of doxorubicin and dacarbazine with or without ifosfamide and mesna in advanced soft tissue and bone sarcomas. J Clin Oncol. 1993;11:1276-85.

5. Jelic S, Kovcin V, Milanovic N, et al. Randomised study of high-dose epirubicin versus high-dose epirubicin-cisplatin chemotherapy for advanced soft tissue sarcoma. Eur J Cancer. 1997;33:220-5.

6. Le Cesne A, Judson I, Crowther D, et al. Randomized phase III study comparing conventional-dose doxorubicin plus ifosfamide versus high-dose doxorubicin plus ifosfamide plus recombinant human granulocyte-macrophage colony-stimulating factor in advanced soft tissue sarcomas: a trial of the European Organization for Research and Treatment of Cancer/Soft Tissue and Bone Sarcoma Group. J Clin Oncol. 2000;18:2676-84.

7. Bramwell VH, Anderson D, Charette ML. Doxorubicin-based chemotherapy for the palliative treatment of adult patients with locally advanced or metastatic soft tissue sarcoma. Cochrane Database Syst Rev. 2003;3:CD003293.

8. Van der Graaf WTA, Blay JY, Chawla SP, Kim DW, Bui-Nguyen B, Casali PG, Schöffski P, Aglietta M, Staddon AP, Beppu Y, et al. Pazopanib for metastatic soft-tissue sarcoma (palette): a randomised, double-blind, placebo-controlled phase 3 trial. Lancet. 2012;379:1879-86.

9. D'Incalci M, Galmarini CM. A review of trabectedin (ET-743): a unique mechanism of action. Mol Cancer Ther. 2010;9:2157-63.

10. Guirouilh-Barbat J, Redon C, Pommier Y. Transcription-coupled DNA double-strand breaks are mediated via the nucleotide excision repair and the mre11-rad50-nbs1 complex. Mol Biol Cell. 2008;19:3969-81.

11. Allavena P, Signorelli M, Chieppa M, Erba E, Bianchi G, Marchesi F, et al. Anti-inflammatory properties of the novel antitumor agent yondelis (trabectedin): inhibition of macrophage differentiation and cytokine production. Cancer Res. 2005;65:2964-71.

12. Germano G, Frapolli R, Simone $M$, Tavecchio $M$, Erba E, Pesce $S$, et al. Antitumor and anti-inflammatory effects of trabectedin on human myxoid liposarcoma cells. Cancer Res. 2010;70:2235-44.
13. D'Incalci M, Badri N, Galmarini CM, Allavena P. Trabectedin, a drug acting on both cancer cells and the tumour microenvironment. $\mathrm{Br} \mathrm{J}$ Cancer. 2014;111:646-50.

14. Reichardt P. Beyond clinical trials in advanced soft tissue sarcoma: what to expect from trabectedin treatment? Futur Oncol. 2015;11(11 s):1.

15. Van Kesteren C, Cvitkovic E, Taamma A, López-Lázaro L, Jimeno JM, Guzman C, Mathôt RAA, Schellens JHM, Misset JL, Brain E, et al. Pharmacokinetics and pharmacodynamics of the novel marine-derived anticancer agent ecteinascidin 743 in a phase I dose-finding study. Clin Cancer Res. 2000;6:4725-32.

16. Delaloge S, Yovine A, Taamma A, Riofrio M, Brain E, Raymond E, Cottu P, Goldwasser F, Jimeno J, Misset JL, Marty M, Cvitkovic E. Ecteinascidin-743: a marine-derived compound in advanced, pretreated sarcoma patients-preliminary evidence of activity. J Clin Oncol. 2001;19:1248-55.

17. Taamma A, Misset JL, Riofrio M, Guzman C, Brain E, Lopez-Lazaro L, Rosing H, Jimeno JM, Cvitkovic E. Phase I and pharmacokinetic study of ecteinascidin-743, a new marine compound, administered as a 24-h continuous infusion in patients with solid tumors. J Clin Oncol. 2001;19:1256-65.

18. Ryan DP, Supko JG, Eder JP, Seiden MV, Demetri G, Lynch TJ, Fischman AJ, Davis J, Jimeno J, Clark JW. Phase I and pharmacokinetic study of ecteinascidin 743 administered as a 72-h continuous intravenous infusion in patients with solid malignancies. Clin Cancer Res. 2001;7:231-42.

19. Villalona-Calero MA, Eckhardt SG, Weiss G, Hidalgo M, Beijnen JH, van Kesteren C, Rosing H, Campbell E, Kraynak M, Lopez-Lazaro L, et al. A phase I and pharmacokinetic study of ecteinascidin-743 on a daily $\times 5$ schedule in patients with solid malignancies. Clin Cancer Res. 2002;8:75-85.

20. Twelves C, Hoekman K, Bowman A, Vermorken JB, Anthoney A, Smyth J, van Kesteren C, Beijnen JH, Uiters J, Wanders J, et al. Phase I and pharmacokinetic study of Yondelis $^{\mathrm{TM}}$ (ecteinascidin-743; et-743) administered as an infusion over $1 \mathrm{~h}$ or $3 \mathrm{~h}$ every 21 days in patients with solid tumours. Eur J Cancer. 2003;39:1842-51.

21. Forouzesh B, Hidalgo M, Chu Q, Mita A, Mita M, Schwartz G, et al. Phase I and pharmacokinetic study of trabectedin as a 1- or 3-h infusion weekly in patients with advanced solid malignancies. Clin Cancer Res. 2009;15:3591-9. 
22. Blay JY, von Mehren M, Samuels BL, Fanucchi MP, Ray-Coquard I, Buckley B, et al. Phase I combination study of trabectedin and doxorubicin in patients with soft tissue sarcoma. Clin Cancer Res. 2008;14:6656-62.

23. Messersmith W, Jimeno A, Ettinger D, Lajeru D, Brahmer J, Lansery D, et al. Phase I trial of weekly trabectedin (et-743) and gemcitabine in patients with advanced solid tumors. Cancer Chemother Pharmacol. 2008;63:181-8.

24. Von Mehren M, Schilder RJ, Cheng JD, Temmer E, Cardoso TM, Renshaw FG, et al. A phase I study of the safety and pharmacokinetics of trabectedin in combination with pegulated liposomal doxorubicin in patients with advanced malignancies. Ann Onco. 2008;19:1802-9.

25. Sessa C, Cresta S, Noberasco C, Capri G, Gallerani E, Braud FD, et al. Phase I clinical and pharmacokinetic study of trabectedin and cisplatin in solid tumours. Eur J Cancer. 2009;45:2116-22.

26. Sessa C, Perotti A, Noberasco C, De Braud F, Gallerani E, Cresta S, et al. Phase I clinical and pharmacokinetic study of trabectedin and doxorubicin in advanced soft tissue sarcoma and breast cancer. Eur J Cancer. 2009;45:1153-61.

27. Perez-Ruixo JJ, Zannikos P, Hirankarn S, et al. Population pharmacokinetic meta-analysis of trabectedin (ET-743, YondelisR) in cancer patients. Clin Pharmacokinet. 2007;46:867-84.

28. Ryan DP, Puchalski T, Supko JG, et al. A phase II and pharmacokinetic study of ecteinascidin 743 in patients with gastrointestinal stromal tumors. Oncologist. 2002;7:531-8.

29. Yovine A, Riofrio M, Blay JY, Brain E, Alexandre J, Kahatt C, et al. Phase II study of ecteinascidin-743 in advanced pretreated soft tissue sarcoma patients. J Clin Oncol. 2004;22:890-9.

30. Garcia-Carbonero R, Supko JG, Manola J, Seiden MV, Harmon D, Ryan DP, et al. Phase II and pharmacokinetic study of ecteinascidin 743 in patients with progressive sarcomas of soft tissues refractory to chemotherapy. J Clin Oncol. 2004;22:1480-90.

31. Blay JY, Le Cesne A, Verweij J, Scurr M, Seynaeve C, Bonvalot $S$, et al. A phase II study of ET-743/trabectedin ('Yondelis') for patients with advanced gastrointestinal stromal tumours. Eur J Cancer. 2004;40:1327-31.

32. Le Cesne A, Blay JY, Judson I, Van Oosterom A, Verweij J, Radford J, et al. Phase ii study of et-743 in advanced soft tissue sarcomas: a european organisation for the research and treatment of cancer (eortc) soft tissue and bone sarcoma group trial. J Clin Oncol. 2005;23:576-84.

33. Morgan JA, Le Cesne A, Chawla S, et al. Randomized phase II study of trabectedin in patients with liposarcoma and leiomyosarcoma (L-sarcomas) after failure of prior anthracylines (A) and ifosfamide (I). ASCO Meeting Abstracts. 2007;25:10060.

34. Monk BJ, Blessing JA, Street DG, Muller CY, Burke JJ, Hensley ML. A phase II evaluation of trabectedin in the treatment of advanced, persistent, or recurrent uterine leiomyosarcoma: A gynecologic oncology group study. Gynecol Oncol. 2012;124:48-52.

35. Gronchi A, Bui BN, Bonvalot S, Pilotti S, Ferrari S, Hohenberger $\mathrm{P}$, et al. Phase II clinical trial of neoadjuvant trabectedin in patients with advanced localized myxoid liposarcoma. Ann Oncol. 2012;23:771-6.

36. Demetri GD, Chawla SP, von Mehren M, Ritch P, Baker LH, Blay JY, et al. Efficacy and safety of trabectedin in patients with advanced or metastatic liposarcoma or leiomyosarcoma after failure of prior anthracyclines and ifosfamide: results of a randomized phase ii study of two different schedules. J Clin Oncol. 2009;27:4188-96.

37. Huygh G, Clement PMJ, Dumez H, Schoffski P, Wildiers $H$, Selleslach J, et al. Ecteinascidin-743: evidence of activity in advanced, pretreated soft tissue and bone sarcoma patients. Sarcoma. 2006; Article ID 56282:1-11.

38. Schöffski P, Taron M, Jimeno J, Grosso F, Sanfilipio $\mathrm{R}$, Casali PG, et al. Predictive impact of DNA repair functionality on clinical outcome of advanced sarcoma patients treated with trabectedin: a retrospective multicentric study. Eur J Cancer. 2011;47:1006-12.

39. Grosso F, Jones RL, Demetri GD, Judson IR, Blay J-Y, Le Cesne A, et al. Efficacy of trabectedin (ecteinascidin-743) in advanced pretreated myxoid liposarcomas: a retrospective study. Lancet Oncol. 2007;8:595-602.

40. Sanfilippo R, Grosso F, Jones RL, Banerjee S, Pilotti $S$, D'Incalci $M$, et al. Trabectedin in advanced uterine leiomyosarcomas: a retrospective case series analysis from two reference centers. Gynecol Oncol. 2011;123:553-6.

41. Le Cesne A, Ray-Coquard I, Duffaud F, Chevreau C, Penel N, Bui Nguyen B, et al. Trabectedin in patients with advanced soft tissue sarcoma: a retrospective national analysis of the French Sarcoma Group. Eur J Cancer. 2015;51:742-50. 
42. Blay JY, Leahy MG, Nguyen BB, Patel SR, Hohenberger P, Santoro A, et al. Randomised phase III trial of trabectedin versus doxorubicin-based chemotherapy as first-line therapy in translocation-related sarcomas. Eur J Cancer. 2014;50:1137-47.

43. Demetri GD, von Mehren M, Jones RL, Hensley ML, Schuetze SM, Staddon A, et al. Efficacy and safety of trabectedin or dacarbazine for metastatic liposarcoma or leiomyosarcoma after failure of conventional chemotherapy: Results of a phase III randomized multicenter clinical trial. J Clin Oncol. 2016;34(8):786-93.

44. Samuels BL, Chawla S, Patel S, von Mehren M, Hamm J, Kaiser PE, et al. Clinical outcomes and safety with trabectedin therapy in patients with advanced soft tissue sarcomas following failure of prior chemotherapy: results of a worldwide expanded access program study. Ann Oncol. 2013;24:1703-9.

45. Prescribing information for Yondelis ${ }^{\circledR}$. http://www. accessdata.fda.gov/drugsatfda_docs/label/2015/207 953s000lbl.pdf. Accessed 26 Apr 2016.

46. Cassier PA, Dufresne A, Blay JV, Fayette J. Trabectedin and its potential in the treatment of soft tissue sarcoma. Ther Clin Risk Management. 2008;4:109-16.
47. Grosso F, Dileo P, Sanfilippo R, et al. Steroid premedication markedly reduces liver and bone marrow toxicity of trabectedin in advanced sarcoma. Eur J Cancer. 2006;42:1484-90.

48. European Medicines Agency. EMA/262441/2015; EMEA/H/C/000773.

49. Syed I, Sudan M, Tseng WW, Arasheben S, Leong B, Aryanfar B, et al. Trabectedin: an active agent in the treatment of advanced mesenchymal chondrosarcoma. Salt Lake City: CTOS Annual Meeting; 2015.

50. Kawai A, Sugiura H, Ueda $T$, Yonemoto $T$, Takahashi M, Morioka $\mathrm{H}$, et al. Trabectedin monotherapy after standard chemotherapy versus best supportive care in patients with advanced, translocation-related sarcoma: a randomized, open-labe; phase 2 study. Lancet Oncol. 2015;16:406-16.

51. http://www.clinicaltrials.gov. Accessed 28 Dec 2015.

52. Blay JV, Casali P, Nieto A, Tanovic A, Le Cesne A. Efficacy and safety of trabectedin as an early treatment for advanced or metastatic liposarcoma and leiomyosarcoma. Future Oncol. 2015;10:59-68. 